\title{
Urinary proteomics of Henoch-Schönlein purpura nephritis in children using liquid chromatography-tandem mass spectrometry
}

\author{
Xiang Fang ${ }^{1,2+}$, Heyan $\mathrm{Wu}^{1 \dagger}$, Mei Lu' ${ }^{1}$, Yan Cao ${ }^{3}$, Ren Wang ${ }^{1}$, Meiqiu Wang ${ }^{1}$, Chunlin Gao ${ }^{1 *}$ and Zhengkun Xia ${ }^{1 *}$ (D)
}

\begin{abstract}
Background: Henoch-Schönlein purpura nephritis (HSPN) is the principal cause of morbidity and mortality in children with Henoch-Schönlein purpura (HSP). However, the criteria for risk assessment currently used is not satisfactory. The urine proteome may provide important clues to indicate the development of HSPN.

Methods: Here, we detected and compared the urine proteome of patients with HSPN and healthy controls by liquid chromatography-tandem mass spectrometry (LC-MS/MS) in the data-independent acquisition (DIA) mode. The differentially expressed proteins were analysed by gene ontology (GO) analysis and Kyoto Encyclopedia of Genes and Genomes (KEGG) analysis. For validation, enzyme-linked immunosorbent assay (ELISA) was used to analyse the selected proteins.

Results: A total of 125 proteins (29 upregulated and 96 downregulated) were found to be differentially expressed in children with HSPN compared with the controls. Forty-one proteins were predicted to have direct interactions. The enriched pathways mainly included focal adhesion, cell adhesion molecules, the PI3K-Akt signalling pathway, ECMreceptor interactions and so on. Cell adhesion related to the pathogenesis of HSPN was the main biological process identified in this study. The decrease in two proteins (integrin beta-1 and tenascin) was validated by ELISA.
\end{abstract}

Conclusions: Our study provides new insights into the assessment of HSPN progression in children, as well as new potential biomarkers. The data confirm the value of the urinary proteome in capturing the emergence of HSPN.

Keywords: Henoch-Schönlein purpura nephritis, Urine, Proteomics, Children, Mass spectrometry

\section{Background}

Henoch-Schönlein purpura (HSP) is the most common systemic vasculitis involving capillaries and the deposition of immunoglobulin A immune complexes in childhood. The annual incidence of HSP varies between 10 and 30 cases per 100000 children $<17$ years old, and most cases occur in children $<10$ years of age [1]. Up to half of

\footnotetext{
*Correspondence: shuangmu34@163.com; njxzk@126.com

${ }^{+}$Xiang Fang and Heyan Wu contributed equally to this work

${ }^{1}$ Department of Pediatrics, Jinling Hospital, the First School of Clinical

Medicine, Southern Medical University, No. 305 Zhongshan East Road, Nanjing 210002, Jiangsu, China

Full list of author information is available at the end of the article
}

children with HSP develop Henoch-Schönlein purpura nephritis (HSPN), 90\% of which occurs within 6 months of onset $[2,3]$. Most patients are diagnosed with HSPN at grade III pathology [4]. Other organ manifestations of HSP are mostly benign and self-limiting, but HSPN may lead to chronic renal disease and end-stage renal failure [5]. Thus, renal involvement may be the principal cause of morbidity and mortality in children with HSP. It is important to clarify the onset mechanism and perform early diagnosis to improve the treatment and prognosis of HSPN. Renal biopsy is the gold standard for detecting renal changes, but it is an invasive examination that cannot be tolerated by most people, and its risks involved do not permit repeated inspection.

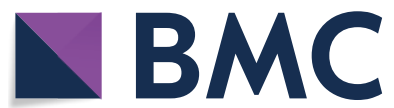

(c) The Author(s) 2020. This article is licensed under a Creative Commons Attribution 4.0 International License, which permits use, sharing, adaptation, distribution and reproduction in any medium or format, as long as you give appropriate credit to the original author(s) and the source, provide a link to the Creative Commons licence, and indicate if changes were made. The images or other third party material in this article are included in the article's Creative Commons licence, unless indicated otherwise in a credit line to the material. If material is not included in the article's Creative Commons licence and your intended use is not permitted by statutory regulation or exceeds the permitted use, you will need to obtain permission directly from the copyright holder. To view a copy of this licence, visit http://creativeco mmons.org/licenses/by/4.0/. The Creative Commons Public Domain Dedication waiver (http://creativecommons.org/publicdomain/ zero/1.0/) applies to the data made available in this article, unless otherwise stated in a credit line to the data. 
In recent years, urine has been considered a potential source to provide important clues regarding the progression of diseases [6]. The normal excretion of proteins in urine is less than $150 \mathrm{mg} / \mathrm{L}$ per $24 \mathrm{~h}$, and approximately $30 \%$ of urinary protein originates from plasma via blood filtration, whereas the remaining $70 \%$ is derived from the kidneys. In patients with HSPN, the filtration barrier is injured, leading to a massive loss of proteins in urine (proteinuria). Urinary proteomics as a novel tool has become an efficient approach for predicting renal involvement prior to renal biopsy and biomarker discovery in kidney diseases [7]. Liquid chromatography-tandem mass spectrometry (LC-MS/MS) is a noninvasive detection method used in urine proteomics that can provide qualitative and quantitative assessments of the proteome and offer clues for biological processes in systems. LC-MS/MS has been applied in the proteomic analysis of various kidney diseases, such as membranous nephropathy [8], acute kidney injury [9], lupus nephritis [10] and IgA nephropathy (IgAN) [11]. Data-independent acquisition (DIA) by LC-MS/MS, which enables comprehensive mapping of the urinary proteome, has sufficient throughput to perform biomarker discovery studies, as all identified proteins can be precisely quantified in hundreds of samples [12].

However, there are few reports of urinary protein alterations focusing on the risk of childhood HSPN. HSPN is characterized by mesangial IgA deposits that induce mesangial cell proliferation and extracellular matrix expansion [13, 14], which are the most common and major pathological change of paediatric HSPN. Some children even have segmental glomerulosclerosis or crescent formation with a loss of functional nephrons in progressive HSPN [13]. The process invariably ends with global glomerular sclerosis under the action of inflammatory mediators. We believe that the roles played by the proteins are worth discussing. In the current study, we aimed to identify the biological significance of differentially expressed proteins from paediatric patients with HSPN via LC-MS/MS in the DIA mode. We expected that some of the differentially expressed proteins could indicate the biological processes involved in the development of HSPN and that the proteomic profile might provide some hint for the treatment and prognosis of HSPN in children.

\section{Materials and methods}

\section{Patients and urine samples}

Midstream morning urine samples were collected from patients with biopsy-proven HSPN (Group A, $\mathrm{n}=34$ ) visiting the Department of Pediatrics, Jinling Hospital, Nanjing, China, from July 2018 to October 2019 (Additional file 1). The enrolment criteria were as follows: (1) age from 2 to 16 years; (2) abnormal urinalysis, including hematuria and/or proteinuria, within 6 months of HSP or renal biopsy results of mesangial proliferative glomerulonephritis characterized by an IgA mesangial deposition [15]; and (3) cases with congenital or hereditary diseases and with severe cardiopulmonary diseases were excluded from the present study. Early-morning spot urine from healthy volunteers (Group B, $\mathrm{n}=33$ ) and HSP children (Group C, $\mathrm{n}=10$ ) was also collected (Additional files 2 and 3). Since HSPN originates from HSP in children, urine samples from HSP children were collected for further validation to exclude possible false positives. The baseline characteristics of the participants are shown in Table 1. Among them, urine samples from group A and group B (4 children in each group) were detected using the DIA method via LC-MS/MS. Thirty samples from group A, 29 from group $\mathrm{B}$ and 10 from group $\mathrm{C}$ were incorporated into the validation cohort. Samples were kept at room temperature for less than $4 \mathrm{~h}$, followed by low-speed centrifugation at $2000 \mathrm{~g}$ for $10 \mathrm{~min}$ at room temperature to remove cellular debris and were then stored at $-80^{\circ} \mathrm{C}$ until use. The study was approved by the Ethics Committee of Jinling Hospital (2019nzgkj-159).

\section{Protein preparation and digestion}

Equal volume samples were concentrated to the same volume by ultrafiltration. Lysis buffer (4\% SDS, $100 \mathrm{mM}$ DTT, $150 \mathrm{mM}$ Tris- $\mathrm{HCl} \mathrm{pH}$ 8.0) was added to the samples directly. The lysates were boiled for 15 min using a homogenizer (Fastprep ${ }^{\circledR}$-24, MP Biomedical, USA) and broken by ultrasound for $3 \mathrm{~min}$. After centrifugation at $14,000 \mathrm{~g}$ for $40 \mathrm{~min}$, the supernatant was quantified using the BCA Protein Assay Kit (Bio-Rad, USA).

Protein digestion was performed according to the FASP procedure described by Wisniewski [16]. Briefly, $200 \mu \mathrm{g}$ of protein was incorporated into $30 \mu \mathrm{l}$ SDT buffer (4\% SDS, $100 \mathrm{mM}$ DTT, $150 \mathrm{mM}$ Tris- $\mathrm{HCl} \mathrm{pH}$ 8.0). The detergent, DTT and other low molecular weight components were removed using UA buffer ( $8 \mathrm{M}$ urea, $150 \mathrm{mM}$ Tris $\mathrm{HCl} \mathrm{pH} \mathrm{8.0)} \mathrm{by} \mathrm{repeated} \mathrm{ultrafiltration} \mathrm{(Microcon}$ units, $30 \mathrm{kD}$, Millipore, USA). One hundred microliters of $0.05 \mathrm{M}$ iodoacetamide in UA buffer was added to block reduced cysteine residues. Then, the samples were incubated for $30 \mathrm{~min}$ in the dark. The filters were washed with $100 \mu \mathrm{l}$ UA buffer three times and then $100 \mu \mathrm{l} 25 \mathrm{mM}$ $\mathrm{NH}_{4} \mathrm{HCO}_{3}$ twice. Finally, the protein suspensions were digested with $2 \mu \mathrm{g}$ trypsin (Promega, USA) in $40 \mu \mathrm{l}$ $100 \mathrm{mM} \mathrm{NH}_{4} \mathrm{HCO}_{3}$ buffer overnight at $37^{\circ} \mathrm{C}$. The resulting peptides were collected as a filtrate. The peptide content was estimated by the spectral density of UV light at $280 \mathrm{~nm}$.

Digested pool peptides were then fractionated into 10 fractions using a Thermo Scientific ${ }^{\mathrm{TM}}$ Pierce $^{\mathrm{TM}}$ High $\mathrm{pH}$ 
Table 1 Baseline characteristics of patients for validation

\begin{tabular}{|c|c|c|c|}
\hline Characteristics & HSPN $(n=30)$ & Normal $(n=29)$ & $\operatorname{HSP}(n=10)$ \\
\hline Onset age (years) & $9.4 \pm 2.4$ & $8.8 \pm 2.9$ & $9.3 \pm 2.5$ \\
\hline Male gender n (\%) & $20(66.7 \%)$ & $12(41.4 \%)$ & $6(60 \%)$ \\
\hline \multicolumn{4}{|l|}{ Presenting symptoms n (\%) } \\
\hline Macro or microscopic hematuria & $30(100 \%)$ & - & - \\
\hline Proteinuria & $27(90 \%)$ & - & - \\
\hline Purpuric rash & $30(100 \%)$ & - & $10(100 \%)$ \\
\hline Colicky pain & 13(43.3\%) & - & $6(60 \%)$ \\
\hline Arthralgia & $11(36.7 \%)$ & - & $5(50 \%)$ \\
\hline Edema & - & - & - \\
\hline Bloody stools & - & - & - \\
\hline \multicolumn{4}{|l|}{ Renal pathology n (\%) } \\
\hline IgA mesangial deposition & $30(100 \%)$ & - & - \\
\hline Mesangial proliferation & $30(100 \%)$ & - & - \\
\hline Crescents & $15(50 \%)$ & - & - \\
\hline Segmental glomerulosclerosis & 13(43.3\%) & - & - \\
\hline \multicolumn{4}{|l|}{ Laboratory measurements } \\
\hline Serum IgA levels (g/l) & $1.8 \pm 0.5$ & - & $1.9 \pm 0.3$ \\
\hline Serum IgE levels (IU/ml) & $193.3 \pm 182.9$ & - & $285.3 \pm 106.7$ \\
\hline Serum creatinine $(\mu \mathrm{mol} / \mathrm{l})$ & $36.0 \pm 8.5$ & - & $34.0 \pm 5.4$ \\
\hline eGFR (ml/min/1.73 m²) & $200.7 \pm 31.3$ & - & $203.8 \pm 15.3$ \\
\hline
\end{tabular}

eGFR is estimated by the Schwartz formula [ $\mathrm{K}^{*}$ height $(\mathrm{cm}) /$ serum creatinine $\left.(\mu \mathrm{mol} / \mathrm{L})\right]$

HSPN Henoch-Schönlein purpura nephritis, Normal healthy controls, HSP Henoch-Schönlein purpura, eGFR estimated glomerular filtration rate

Reversed-Phase Peptide Fractionation Kit. Each fraction was concentrated by vacuum centrifugation and reconstituted in $15 \mu \mathrm{l}$ of $0.1 \%(\mathrm{v} / \mathrm{v})$ formic acid. Collected peptides were desalted on C18 Cartridges [Empore ${ }^{\mathrm{TM}}$ SPE Cartridges C18 (standard density), bed I.D. $7 \mathrm{~mm}$, volume $3 \mathrm{ml}$, Sigma]. HRM calibration peptides (Biognosys) were added to the samples before analysis according to the manufacturer's instructions for the DIA experiments.

\section{LC-MS/MS by Q Exactive HF}

MS experiments were performed on a Q Exactive mass spectrometer that was coupled to an Easy nLC 1200 chromatography system (Thermo Scientific, Waltham, MA, USA). Each DIA cycle contained one full MS-SIM scan, and 30 DIA scans covered a mass range from 350 to $1650 \mathrm{~m} / \mathrm{z}$ with the following settings: the SIM full scan resolution was 60,000 at $200 \mathrm{~m} / \mathrm{z}$; AGC, 3e6; maximum IT, $50 \mathrm{~ms}$; and profile mode; the DIA scans were performed at a resolution of 30,000; AGC target, 3e6; Max IT, auto; and normalized collision energy, $30 \mathrm{eV}$. Peptides ( $2 \mu \mathrm{l}$ of digest) were separated by a linear gradient from buffer B ( $80 \%$ acetonitrile and $0.1 \%$ formic acid) at a flow rate of $250 \mathrm{nl} / \mathrm{min}$. The total run time with the loading and washing steps was $120 \mathrm{~min}$. The column oven was set to $40{ }^{\circ} \mathrm{C}$.

\section{Sequence database search and data analysis}

The MS data were analysed using MaxQuant software version 1.5.3.17. The database was downloaded from the website http://www.uniprot.org. iRT peptide sequences were added (>Biognosys|iRT Kit|Sequence_fusion: LGGNEQVTRYILAGVENSKGTFIIDPGGVIRGTFIIDPAAVIRGAGSSEPVTGLDAKTPVISGGPYEYRVEATFGVDESNAKTPVITGAPYEYRDGLDAASYYAPVRADVTPADFSEWSKLFLQFGAQGSPFLK). The parameters were set as follows: the enzyme was trypsin, maximum missing cleavage was 2 , fixed modification was carbamidomethyl (C), and dynamic modification was oxidation (M) and acetyl (Protein $\mathrm{N}$ term). All the reported data were based on the $99 \%$ confidence interval for protein identification as determined by the false discovery rate $(\mathrm{FDR}=\mathrm{N}($ decoy $) * 2 /(\mathrm{N}($ decoy $)+\mathrm{N}($ target $))) \leq 1 \%$. A spectral library was constructed by importing the original raw files. DIA data were analysed with a Spectronaut Pulsar $\mathrm{X}^{\mathrm{TM}}$ search of the above constructed spectral library. All the results were filtered based on a $\mathrm{Q}$ value cutoff of 0.01 (equivalent to FDR $<1 \%$ ).

Protein intensity was calculated by summing the peptide peak areas of each protein from the Spectronaut output file. To test the spectral libraries, one urinary sample was measured three times using the Q Exactive HF and was then analysed in Spectronaut. The results were 
benchmarked based on the numbers of detected peptides and proteins as well as the reproducibility of the peptide and protein detection. For the statistical and bioinformatic analysis, only the urinary proteins present in at least 3 patients in the same group were used.

\section{Bioinformatics analyses}

The proteins were defined as differentially expressed if the fold-change of comparing the disease groups and healthy controls was $\geq 2$ or $\leq 0.5$ and the $P$ value $<0.05$. The differentially expressed proteins were analysed by hierarchical clustering to classify all samples (http:// en.wikipedia.org/wiki/Cluster_analysis). Next, the differentially expressed proteins were subjected to gene ontology (GO) analysis by Blast2GO (https://www.blast 2go.com/) and matched against the Kyoto Encyclopedia of Genes and Genomes (KEGG) database by the KEGG Automatic Annotation Server (KAAS, https://www. genome.jp/tools/kaas/). $\mathrm{P}<0.05$ in Fisher's exact test was considered significant. Protein-protein interaction (PPI) networks were created for these proteins using the STRING database (http://string-db.org/).

\section{Validation by ELISA analysis}

All urinary samples were thawed, centrifuged and aliquoted according to the manufacturer's instructions for ELISA kits (CUSABIO Biotech CO., LTD). Then, $100 \mu \mathrm{l}$ of a biotin-conjugated antibody (1:100 dilution) and $100 \mu \mathrm{l}$ of horseradish peroxidase (HRP)-avidin (1:100 dilution) were added to $100 \mu \mathrm{l}$ of each sample after centrifugation at 10,000 $\mathrm{g}$ for $15 \mathrm{~min}$. After development with the TMB substrate solution, the reaction was terminated by adding $50 \mu \mathrm{l}$ of the stop solution. The optical density (OD) values were read at $450 \mathrm{~nm}$, and the concentrations were automatically calculated according to the standard curve.

\section{Statistical analysis}

We used SPSS software version 19.0 for Windows (IBM, Chicago, IL, USA) for statistical analyses. The data were subjected to the Shapiro-Wilk normality test. Continuous variables are expressed as means \pm standard deviations. Categorical variables are expressed as absolute numbers and percentages (\%). The significance of the protein abundance changes was calculated using nonparametric Student's $t$ test, and Bonferroni multiple testing correction was applied. A two-tailed test with $\mathrm{p}<0.05$ was considered significant. Graphs were prepared using GraphPad Prism version 7.0 (GraphPad Software, San Diego, CA, USA).

\section{Results}

\section{Integrated proteome information}

A total of 1209 non-redundant proteins were detected in urine from HSPN patients, and 125 proteins (29 upregulated and 96 downregulated) were found to be differentially expressed in HSPN patients compared with those from healthy controls (Additional file 4). Hierarchical clustering analysis was conducted on 125 dysregulated proteins, and the heatmap obtained from the analysis provided protein profiles across the HSPN group and healthy controls (Fig. 1).

\section{GO functional analysis}

We performed GO analysis to enrich and cluster the 125 differentially expressed proteins. The detailed information of the molecular functions, cellular components and biological processes is shown in Additional file 5. It was found that most of the proteins were involved in cell adhesion $(n=23)$, which as the main biological process identified by $\mathrm{GO}$ enrichment analysis $(\mathrm{p}<0.05)$. In addition, GO annotation analysis revealed that the proteins were mainly distributed in the intrinsic components of the membrane $(n=46)$ and were mainly associated with the extracellular matrix $(\mathrm{n}=8)$ (Fig. 2).

\section{KEGG pathway analysis}

We next used KEGG analysis to search for enriched pathways including the 125 differential proteins. The detailed information of the KEGG pathway enrichment is shown in Additional file 6. According to the KEGG function classification analysis, focal adhesion, cell adhesion molecules, the phosphatidylinositide 3-kinase-protein kinase B (PI3K-Akt) signalling pathway, extracellular matrix (ECM)-receptor interactions, platelet activation, complement and coagulation cascades, cholesterol metabolism, and regulation of the actin cytoskeleton were among the top 20 enriched pathways (Fig. 3). The aldosterone synthesis and secretion pathway $(\mathrm{p}<0.05)$ was determined to be significantly enriched according to KEGG analysis (Fig. 4).

\section{Proteins interaction analysis}

A total of 41 proteins were predicted to have direct interactions among the 125 differentially expressed proteins according to PPI analysis. Interestingly, the core proteins (integrin beta-1 and tenascin) were involved in multiple pathways, such as focal adhesion, cell adhesion molecules, ECM-receptor interactions and the PI3K-Akt signalling pathway (Fig. 5). In addition, it was found from the network that these proteins were directly or indirectly related to collagen, fibronectin, 


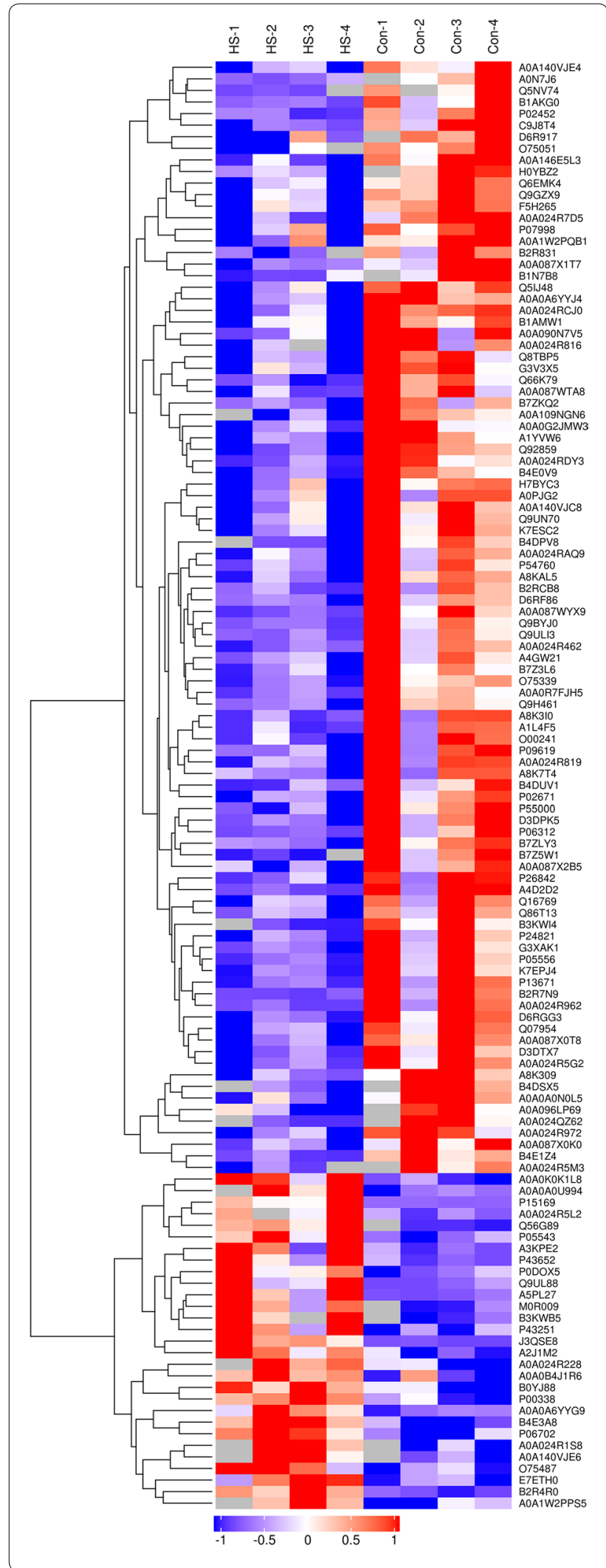

Fig. 1 Cluster analysis of 125 differentially expressed proteins. The hierarchical clustering results are represented as a tree heat map, with the ordinate representing significantly differentially expressed proteins and the abscissa representing sample information. Significant differences in protein expression in the different numerical expression quantities (log2 expression) of the samples with different colours are shown in the heat map, where red represents significantly upregulated proteins, green represents significantly downregulated proteins, and grey represents no quantitative information for proteins. HS-1, 2, 3, and 4 represent patients with Henoch-Schönlein purpura nephritis, and Con-1, 2, 3, and 4 represent the healthy control group

and fibrinogen. Therefore, integrin beta- 1 and tenascin were selected for further validation.

\section{ELISA analysis}

To validate the results of the proteomic analysis, two proteins (integrin beta-1 and tenascin) were chosen for further study, and their urinary levels in the validation cohort, including 30 HSPNs, 29 healthy controls, and 10 HSPs, were measured by using ELISA kits. The urinary levels results are shown in Fig. 6a, b. Integrin beta-1 and tenascin were significantly downregulated in HSPN patients compared to healthy controls $(p<0.05)$, but compared with HSP, integrin beta-1 had no significant difference $(p=0.508)$, while tenascin had a significant difference $(p=0.005)$ in HSPN.

\section{Discussion}

Currently, renal involvement is critical for the prognosis of children with HSPN. Most of the need lies in the detection of HSPN patients at risk of occurrence and the progression of renal disease, and early diagnosis and treatment may greatly contribute to enhancing the survival of children. Monitoring changes in the urinary proteome may have an advantage in the diagnosis of kidney disease. The review of Julian BA et al. concluded urinary detection is more sensitive than current standard clinical testing and far less risky than renal biopsy, and so provides a useful tool for the diagnosis and monitoring of patients with IgA associated renal diseases [17]. Targeted proteomics is a hot research topic that can identify promising biomarkers of disease activity and organ involvement [18]. However, proteomic analysis of HSPN has rarely been studied, especially in urine. He XL et al. performed a comparative analysis of serum proteomes by LC-MS/MS that revealed proteomic alterations in HSPN patients [19]. An analysis of urinary polypeptides from HSPN patients by electrophoretic methods detected urine proteins such as collagen alpha-1 (I) and (III) and alpha-1-antitrypsin [20]. To the best of our knowledge, 


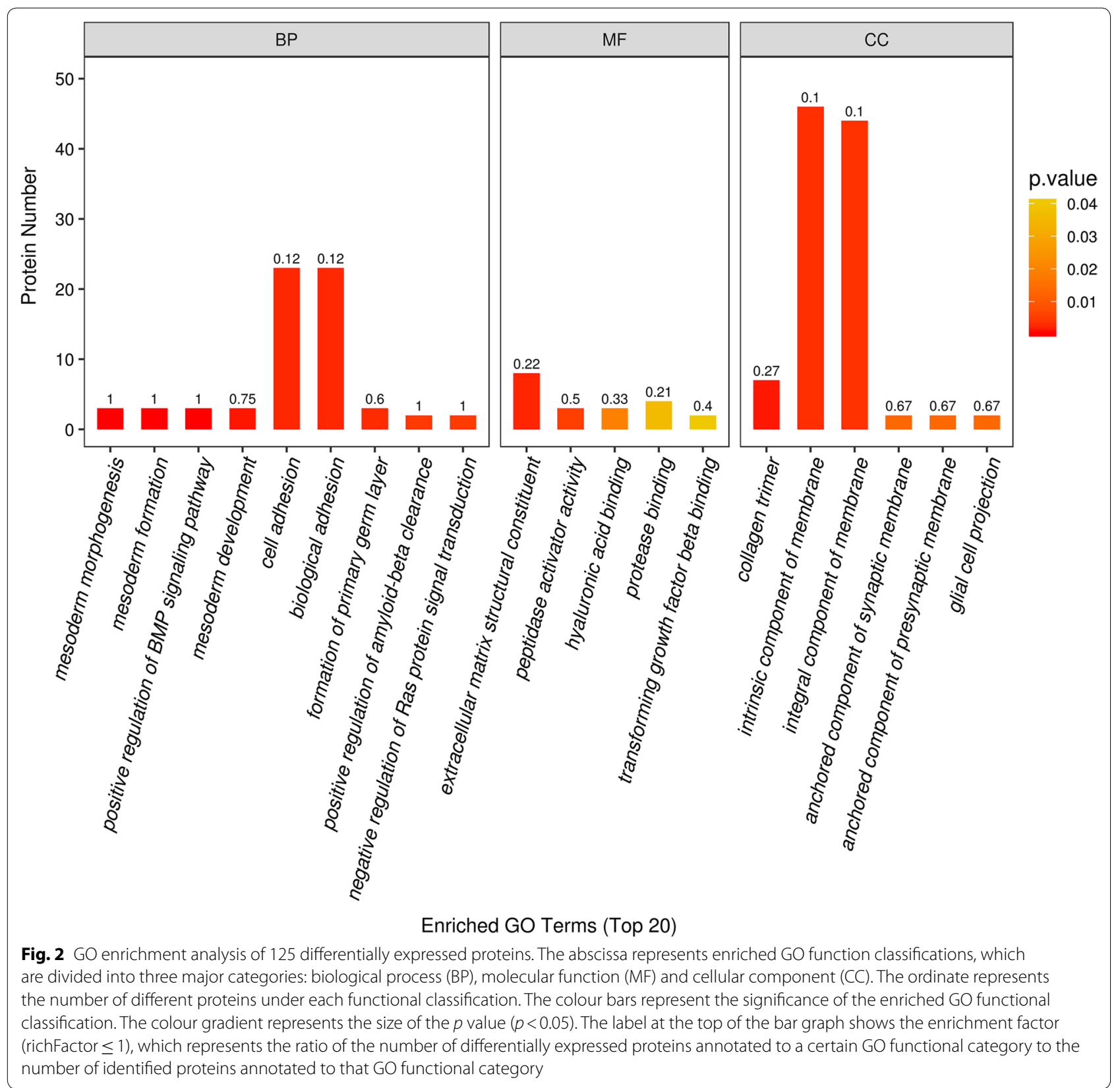

no proteomic analysis of urine has been reported for the discovery of biomarkers and/or to describe the involvement of related biological pathways in children with HSPN. In the present study, we performed a comparative analysis of the urinary proteome to obtain insights into the pathogenesis in HSPN children using the DIA method via LC-MS/MS. As a result, we found that 125 proteins were differentially expressed in urine form HSPN patients compared to that from healthy controls. Forty-one proteins were predicted to directly interact with each other.
According to GO annotation, our study found that most of the differentially expressed proteins are involved in cell adhesion. Cell adhesion linking cells to the ECM is crucial for maintaining the mechanical integrity of podocytes, which is a key component of the glomerular filtration barrier [21], and integrin beta- 1 is the major cell-matrix adhesion receptor in podocytes, which connects laminin in GBM through various adaptor proteins to the intracellular actin cytoskeleton [22]. This process may be critical for the development of HSPN in children. According to KEGG function classification analysis, a 

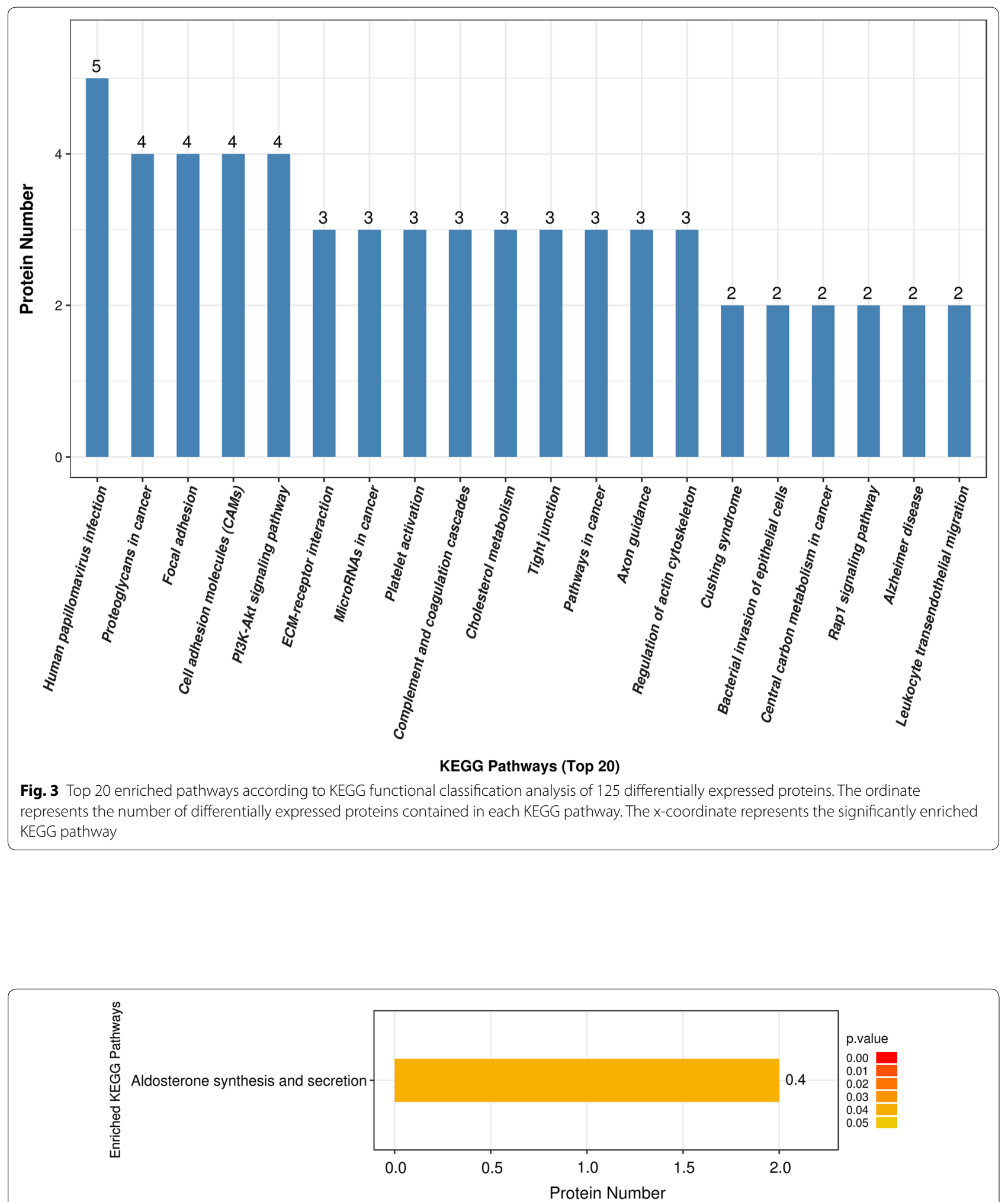

Fig. 4 KEGG pathway enrichment analysis of 125 differentially expressed proteins. The ordinate represents the significantly enriched KEGG pathway. The $x$-coordinate represents the number of differentially expressed proteins contained in each KEGG pathway. The colour bars represent the significance of the enriched KEGG pathways. The colour gradient represents the size of $p$ values $(p<0.05)$. The label at the top of the bar graph shows the enrichment factor (richFactor $\leq 1$ ), which represents the proportion of the number of differentially expressed proteins involved in a KEGG pathway among all identified proteins 


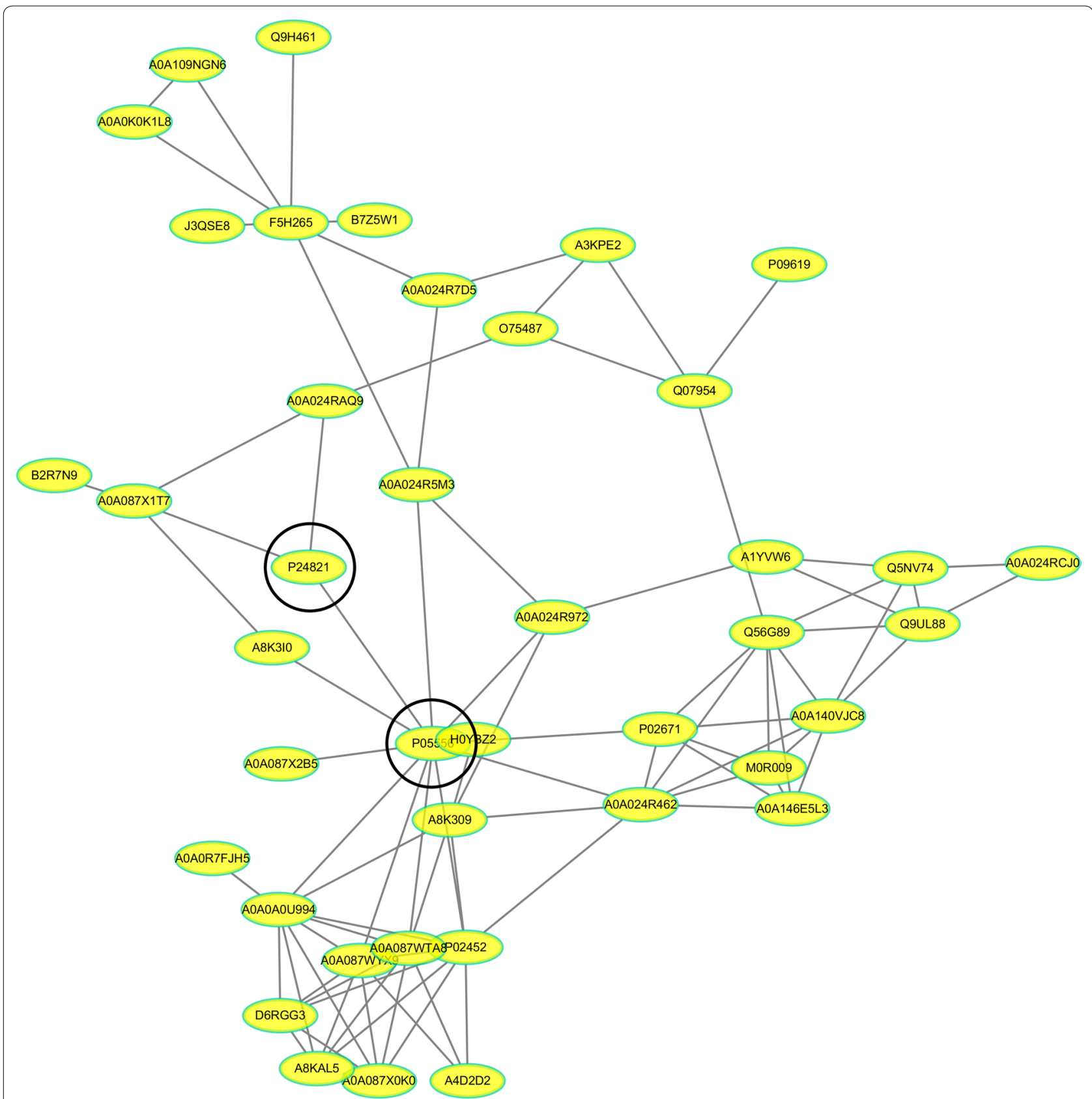

Fig. 5 Differentially expressed protein interaction networks. In the protein interaction network, nodes represent proteins and lines represent protein-protein interactions. Yellow nodes are differentially expressed proteins. The number of proteins directly interacting with protein A is called the linkage degree of protein A. P24821 tenascin, P05556 integrin beta-1, A0A024RAQ9 versican, P02452 collagen alpha-1 (I), P02671 Fibrinogen alpha, A0A024R462 fibronectin-1

variety of enriched pathways are involved in HSPN, such as focal adhesion, cell adhesion molecules, the PI3KAkt signalling pathway, ECM-receptor interactions and regulation of the actin cytoskeleton, which include many common differentially expressed proteins (integrin beta1 , tenascin, collagen alpha-1 (I) and platelet-derived growth factor receptor) that participate in mesangial cell proliferation and fibrosis processes [23-25]. HSPN is driven by mesangial IgA deposits and the resulting mesangial cell proliferation and glomerular segmental sclerosis according to our renal biopsy results. The 

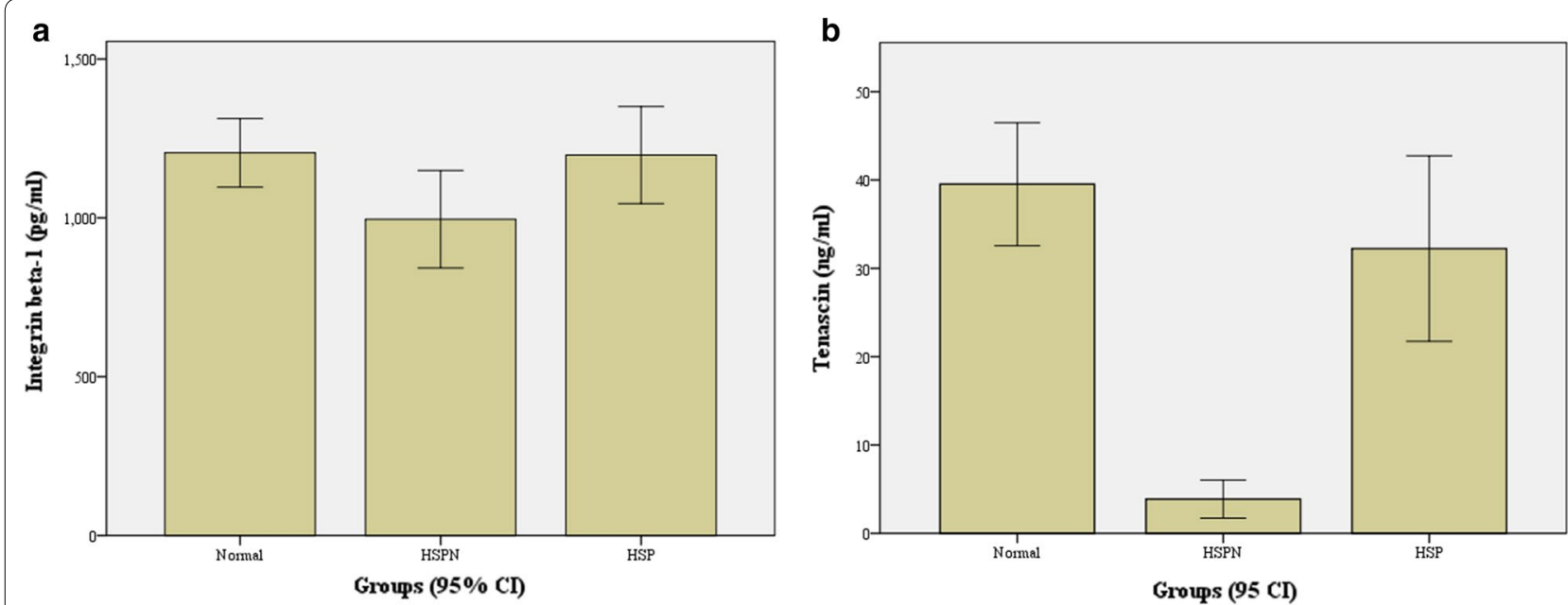

Fig. 6 Urinary levels of integrin beta-1 and tenascin by ELISA in the validation cohort. a The urine concentration level of integrin beta-1 using ELISA kits. $\mathbf{b}$ The urine concentration level of tenascin using ELISA kits. The ordinate represents the concentration of proteins. The x-coordinate represents the group of validation. HSPN represents the group of Henoch-Schönlein purpura nephritis, Normal represents the healthy control group, and HSP represents the group of Henoch-Schönlein purpura

above-mentioned enriched pathways may account in part for the pathological changes associated with HSPN. In addition, our data showed that complement and coagulation cascades and platelet activation are predominantly involved in the pathway analysis. Several studies have reported that the activation of platelets and coagulationcomplement crosstalk may be associated with a higher risk of thrombotic events [26]. HSPN is the pathological type of nephrotic syndrome associated with the highest incidence of thromboembolic events. Similarly, changes occur in aldosterone synthesis and secretion and cholesterol metabolism.

To validate the proteomic analysis results, the downregulation of the two core proteins by ELISA (integrin beta- 1 and tenascin) was found to be consistent with the mass spectrometry results. However, the urine secretion level of integrin beta-1 in children with HSPN was similar to that of children with HSP, which may be related to the participation of integrin beta-1 in multiple pathways in disease. However, the urine secretion level of tenascin was significantly different among HSPN children and HSP children. Therefore, tenascin might be a novel HSPN-related biomarker that can be used to diagnose early renal damage in children with HSPN. Tenascin, a large oligomeric glycoprotein, is a ubiquitous extracellular matrix protein of human renal tissue under normal and pathological conditions [27]. Tenascin is expressed on the cell surface and plays a critical role in a wide array of biological processes, including the immune response, inflammation, and morphogenesis. Tenascin rapidly responds to inflammation and injury, and urine excretion of tenascin may reduce the fibrogenic response to acute tissue damage. Studies have identified tenascin as a major constituent of the fibrogenic niche that promotes fibroblast proliferation [28]. Ozkan $\mathrm{H}$ et al. indicated tenascin as a potential urine biomarker for acute kidney injury using comparative proteomics [29]. In addition, expression of the tenascin protein may be an indicator of disease chronicity, and mRNA expression of tenascin may be an indicator of disease activity with renal involvement [30]. Thus, it may be worthwhile to further investigate the predictive role of urine tenascin on the progression to HSPN.

This study has several limitations. We only performed LC-MS/MS on a few urine samples, and we only outlined the possible candidate proteins involved. It is necessary to increase the accuracy and reduce the variability of our analyses through further verification in a large sample size. We did not assess other glomerular diseases using biological analysis.

\section{Conclusions}

In summary, our study suggests that 125 differentially expressed proteins and multiple enrichment pathways are associated with the pathogenesis of HSPN in children using the DIA method via LC-MS/MS. The value of urinary proteomics in disease recognition was further confirmed. The discovery of differential proteins lays the foundation for searching for potential biomarkers of HSPN in children. 


\section{Supplementary information}

Supplementary information accompanies this paper at https://doi. org/10.1186/s12014-020-09274-x.

Additional file 1. Clinical characteristics of patients in group A.

Additional file 2. Clinical characteristics of patients in group B.

Additional file 3. Clinical characteristics of patients in group $C$.

Additional file 4. Raw data of the information of 125 proteins.

Additional file 5. The detail information of gene ontology (GO) enrichment analysis.

Additional file 6. The detail information of Kyoto Encyclopedia of Genes and Genomes (KEGG) pathway enrichment analysis.

\section{Abbreviations}

HSPN: Henoch-Schönlein purpura nephritis; HSP: Henoch-Schönlein purpura; IgAN: IgA nephropathy; LC-MS/MS: Liquid chromatography-tandem mass spectrometry; DIA: Data independent acquisition; DDA: Data dependent acquisition; GO: Gene ontology; KEGG: Kyoto Encyclopedia of Genes and Genomes; KAAS: KEGG Automatic Annotation Server; ELISA: Enzyme-linked immunosorbent assay; ECM: Extracellular matrix; PI3K-Akt: Phosphatidylinositide 3-kinase-protein kinase B; FDR: False discovery rate; PPI: Protein-protein interaction; OD: Optical density; HRP: Horseradish peroxidase.

\section{Acknowledgements}

The authors gratefully acknowledge the assistance of Dr. Zhengkun Xia of Jinling Hospital and thank to Shanghai Applied Protein Technology Co., Ltd for expertise in LC-MS/MS analysis.

\section{Authors' contributions}

XF, CLG, ZKX designed experiments; XF, HYW, ML performed experiments and drafted the manuscript; RW, MQW collected the samples of patients; $X F$, HYW, ML, YC performed statistical analysis and prepared figures and tables; All authors contributed to reviewing of each manuscript version. All authors read and approved the final manuscript.

\section{Funding}

This study was funded by the Pediatric Medical Innovation Team of Jiangsu Province (CXTDA2017022), the Primary Research \& Development Plan of Jiangsu Province (BE2017719) and the Projects of Natural Science Research in Colleges and Universities of Anhui Province (KJ2017A884).

\section{Availability of data and materials}

All data generated or analysed during this study are included in this published article and its additional files.

\section{Ethics approval and consent to participate}

This study was approved by the Ethics Committee of Jinling Hospital (2019nzgkj-159) and informed consents were obtained from all participants.

\section{Consent for publication}

Written informed consents for publication were obtained from all participants.

\section{Competing interests}

The authors declare that they have no conflict of interests.

\section{Author details}

${ }^{1}$ Department of Pediatrics, Jinling Hospital, the First School of Clinical Medicine, Southern Medical University, No. 305 Zhongshan East Road, Nanjing 210002, Jiangsu, China. ${ }^{2}$ Department of Clinical Medicine, Anqing Medical College, Anqing 246052, Anhui, China. ${ }^{3}$ Nanjing Maternity and Child Health Care Institute, Women's Hospital of Nanjing Medical University, Nanjing Maternity and Child Health Care Hospital, Nanjing 210004, Jiangsu, China.

Received: 10 November 2019 Accepted: 27 February 2020

Published online: 12 March 2020

\section{References}

1. Trnka Peter. Henoch-Schönlein purpura in children. J Paediatr Child Health. 2013:49:995-1003.

2. Chen JY, Mao JH. Henoch-Schönlein purpura nephritis in children: incidence, pathogenesis and management. World J Pediatr. 2015;11:29-34.

3. Narchi H. Risk of long term renal impairment and duration of follow up recommended for Henoch-Schonlein purpura with normal or minimal urinary findings: a systematic review. Arch Dis Child. 2005;90:916-20.

4. Feng D, Huang WY, Hao S, Niu XL, Wang P, Wu Y, et al. A single-center analysis of Henoch-Schonlein purpura nephritis with nephrotic proteinuria in children. Pediatr Rheumatol Online J. 2017;15:15.

5. Pohl M. Henoch-Schönlein purpura nephritis. Pediatr Nephrol. 2015;30:245-52

6. Leng W, Ni X, Sun C, Lu T, Malovannaya A, Jung SY, et al. Proof-of-concept workflow for establishing reference intervals of human urine proteome for monitoring physiological and pathological changes. EBioMedicine. 2017;18:300-10

7. Wu J, Chen YD, Gu W. Urinary proteomics as a novel tool for biomarker discovery in kidney diseases. J Zhejiang Univ Sci B. 2010;11:227-37.

8. Pang L, Li Q, Li Y, Liu Y, Duan N, Li H. Urine proteomics of primary membranous nephropathy using nanoscale liquid chromatography tandem mass spectrometry analysis. Clin Proteomics. 2018;15:5.

9. Malagrino PA, Venturini G, Yogi PS, Dariolli R, Padilha K, Kiers B, et al. Proteome analysis of acute kidney injury-discovery of new predominantly renal candidates for biomarker of kidney disease. J Proteomics. 2017;151:66-73.

10. Caster DJ, Korte EA, Merchant ML, Klein JB, Wilkey DW, Rovin BH, et al. Autoantibodies targeting glomerular annexin A2 identify patients with proliferative lupus nephritis. Proteomics Clin Appl. 2015;9:1012-20.

11. Kalantari S, Rutishauser D, Samavat S, Nafar M, Mahmudieh L, Rezaei-Tavirani $M$, et al. Urinary prognostic biomarkers and classification of IgA nephropathy by high resolution mass spectrometry coupled with liquid chromatography. PLOS ONE. 2013;8:e80830.

12. Muntel J, Xuan Y, Berger ST, Reiter L, Bachur R, Kentsis A, et al. Advancing urinary protein biomarker discovery by data-independent acquisition on a quadrupole-orbitrap massspectrometer. J Proteome Res. 2015;14:4752-62.

13. Huang X, Ma L, Ren P, Wang H, Chen L, Han H, et al. Updated Oxford classification and the international study of kidney disease in children classification: application in predicting outcome of Henoch-Schönlein purpura nephritis. Diagn Pathol. 2019;14:40

14. Nakazawa S, Imamura R, Kawamura M, Kato T, Abe T, Iwatani H, et al. Evaluation of IgA1 o-glycosylation in Henoch-Schönlein purpura nephritis using mass spectrometry. Transplant Proc. 2019;51:1481-7.

15. Zhu CH, Huang SM. Evidence-based guidelines on diagnosis and treatment of Henoch-Schonlein purpura nephritis (2016) interpretation. Chin J Pediatr. 2017; 55:654-7.

16. Wiśniewski JR, Zougman A, Nagaraj N, Mann M. Universal sample preparation method for proteome analysis. Nat Methods. 2009;6:359-62.

17. Julian BA, Wittke S, Haubitz M, Zürbig P, Schiffer E, McGuire BM, et al. Urinary biomarkers of IgA nephropathy and other lgA-associated renal diseases. World J Urol. 2007;25:467-76.

18. Schanstra JP, Mischak H. Proteomic urinary biomarker approach in renal disease: from discovery to implementation. Pediatr Nephrol. 2015;30:713-25.

19. He XL, Yin W, Ding Y, Cui SL, Luan JW, Zhao PW, et al. Higher serum angiotensinogen is an indicator of IgA vasculitis with nephritis revealed by comparative proteomes analysis. PLOS ONE. 2015;10:e0130536.

20. Julian BA, Wittke S, Novak J, Good DM, Coon JJ, Kellmann M, et al. Electrophoretic methods for analysis of urinary polypeptides in IgA-associated renal diseases. Electrophoresis. 2007;28:4469-83.

21. Bachir Al, Horwitz AR, Nelson WJ, Bianchini JM. Actin-based adhesion modules mediate cell interactions with the extracellular matrix and neighboring cells. Cold Spring Harb Perspect Biol. 2017;9:a023234.

22. Sachs N, Sonnenberg A. Cell-matrix adhesion of podocytes in physiology and disease. Nat Rev Nephrol. 2013;9:200-10.

23. Rabb HA. Cell adhesion molecules and the kidney. Am J Kidney Dis. 1994;23:155-66

24. Xiang $\mathrm{H}$, Xue W, Wu X, Zheng J, Ding C, Li Y, et al. FOXP1 inhibits high glucose-induced ECM accumulation and oxidative stress in mesangial cells. Chem Biol Interact. 2019;313:108818.

25. Feng X, Wu C, Yang M, Liu Q, Li H, Liu J, et al. Role of PI3K/Akt signal pathway on proliferation of mesangial cell induced by HMGB1. Tissue Cell. 2016:48:121-5. 
26. Foley JH. Examining coagulation-complement crosstalk: complement activation and thrombosis. Thromb Res. 2016;141(Suppl 2):S50-4.

27. Truong LD, Foster SV, Barrios R, D'Agati V, Verani RR, Gonzalez JM, et al. Tenascin is an ubiquitous extracellular matrix protein of human renal interstitium in normal and pathologic conditions. Nephron. 1996;72:579-86.

28. Fu H, Tian Y, Zhou L, Zhou D, Tan RJ, Stolz DB, et al. Tenascin-C is a major component of the fibrogenic niche in kidney fibrosis. J Am Soc Nephrol. 2017;28:785-801.

29. Ozkan H, Okuturlar Y, Koçoğlu H, Hursitoglu M, Gedikbasi A, Utku IK, et al. Serum levels and urinary excretion of tenascin-C and TIMP-1 in acute kidney injury. Clin Lab. 2019. https://doi.org/10.7754/Clin.Lab.2019.190233.
30. Masaki T, Yorioka N, Taniguchi Y, Oda H, Yamakido M. Tenascin expression may reflect the activity and chronicity of human IgA nephropathy. Clin Nephrol. 1998:50:205-13.

\section{Publisher's Note}

Springer Nature remains neutral with regard to jurisdictional claims in published maps and institutional affiliations.
Ready to submit your research? Choose BMC and benefit from:

- fast, convenient online submission

- thorough peer review by experienced researchers in your field

- rapid publication on acceptance

- support for research data, including large and complex data types

- gold Open Access which fosters wider collaboration and increased citations

- maximum visibility for your research: over $100 \mathrm{M}$ website views per year

At BMC, research is always in progress.

Learn more biomedcentral.com/submissions 